\title{
Incipient non-arteritic anterior ischaemic optic neuropathy: a distinct clinical entity, the Singapore scene 3
}

Daniel $\underline{\text { Chua }}^{1}$, MBBS, James $F \underline{\text { Cullen }}^{1}$, FRCS

\begin{abstract}
We report five cases of non-arteritic anterior ischaemic optic neuropathy (NA-AION) where spontaneous resolution of the optic disc swelling occurred, and all relevant visual modalities were normal at presentation and remained so until resolution of the process after a median time of 9.6 weeks. This condition, which can be termed 'incipient NA-AION' or 'threatened NA-AION', should be recognised so that unnecessary investigations for other and more serious causes of optic disc swelling can be prevented.
\end{abstract}

Keywords: anterior ischaemic optic neuropathy, spontaneous resolution, visual acuity, visual fields

\section{INTRODUCTION}

As previously reported, ${ }^{(1)}$ non-arteritic anterior ischaemic optic neuropathy (NA-AION) is the most common optic neuropathy encountered in Singapore, and it is usually associated with diabetes mellitus, hypertension and hyperlipidaemia. The clinical diagnosis of NA-AION is made based on the presence of a swollen optic nerve head, and in particular a visual field defect, $75 \%$ of which involve the lower fields. ${ }^{(2)}$ There are, however, some cases of NA-AION that are asymptomatic and where all relevant visual modalities are normal. The optic nerve head is similarly swollen, and this may persist for some time with eventual spontaneous resolution. This condition has been termed 'incipient NA-AION' and according to an American report, 25\% of patients with this type of NA-AION eventually progress to full-blown NA-AION after a median time of 5.8 weeks. ${ }^{(3)}$ Such progression, however, has not been observed in Singapore to date. Furthermore, unlike the aforementioned American report, ${ }^{(3)}$ only one of the five patients in the present case series had diabetes mellitus.

\section{CASE REPORTS}

The demographics, medical conditions and resolution time of the five patients who presented to our institution and were diagnosed with the so-called incipient NA-AION over nine months are shown in Table I. Resolution time refers to the duration from the date of presentation or diagnosis in the senior author's (JFC) neuro-ophthalmology clinic at Singapore National Eye Centre, to the date when the optic disc swelling was recorded to have fully resolved.

It must be emphasised that the diagnosis of full-blown or classical NA-AION can only be made in the presence of a visual field defect, even if visual acuity is normal. The diagnosis of incipient NA-AION, however, has to be made based on the presence of optic disc swelling with full visual fields and normal acuity, unless there is another cause such as maculopathy, as was observed in Case 4. The disc swelling that occurs in incipient NA-AION is usually of a mild degree at the outset and resolves spontaneously. In the present case series, the median resolution time was 9.6 weeks.

In the current case series, visual acuity, colour vision and intraocular pressure were normal, except in one diabetic patient with maculopathy (Case 4). In all the cases, the fellow eye was intact. The hallmark sign of optic nerve disease, namely a relative afferent pupil defect (RAPD), was detected in only two patients. This could be due to the presence of only a very mild optic disc oedema and normal visual function at presentation among the remaining three patients.

\section{Case 1}

A 51-year-old Singaporean Malay woman presented to our clinic on March 13, 2013, with a complaint of blurred vision in the left eye $(V L=6 / 7.5)$ that persisted for two weeks. She had a known history of ischaemic heart disease, hypertension

Table I. Demographics, medical condition and resolution time of the five patients with incipient non-arteritic anterior ischaemic optic neuropathy.

\begin{tabular}{|c|c|c|c|c|c|c|c|c|}
\hline \multirow{2}{*}{$\begin{array}{l}\text { Case } \\
\text { no. }\end{array}$} & \multirow[t]{2}{*}{ Age } & \multirow[t]{2}{*}{ Race } & \multirow[t]{2}{*}{ Gender } & \multicolumn{4}{|c|}{ Medical condition } & \multirow{2}{*}{$\begin{array}{l}\text { Resolution } \\
\text { time (wk) }\end{array}$} \\
\hline & & & & Diabetes mellitus & Hypertension & Hyperlipidaemia & Ischaemic heart disease & \\
\hline 1 & 51 & Malay & Female & No & Yes & Yes & Yes & 5 \\
\hline 2 & 56 & Chinese & Female & No & Yes & No & No & 12 \\
\hline 3 & 42 & Chinese & Male & No & No & No & No & 13 \\
\hline 4 & 63 & Chinese & Female & Yes & Yes & No & No & 10 \\
\hline 5 & 60 & Chinese & Female & No & Yes & No & No & 8 \\
\hline
\end{tabular}

${ }^{1}$ Neuro-Ophthalmology Service, Singapore National Eye Centre, Singapore

Correspondence: Dr James F Cullen, Senior Consultant, Neuro-Ophthalmology Service, Singapore National Eye Centre, 11 Third Hospital Avenue, Singapore 168751. jbarrycullen@yahoo.com 

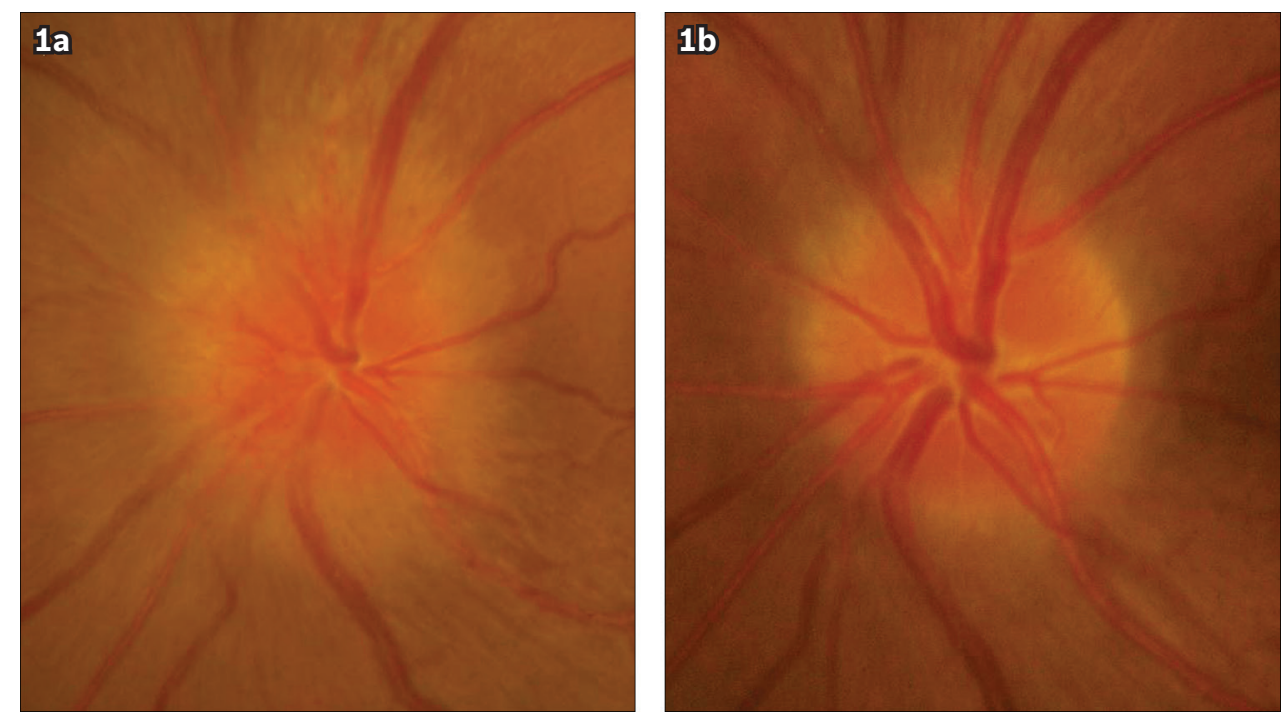

Fig. 1 Fundus photographs show (a) a small hyperaemic swollen optic disc and no haemorrhage; and (b) resolution of the swelling in the same optic disc, with no observable atrophy, three months later.

and hyperlipidaemia, and was receiving treatment for these conditions. On examination, she had no RAPD, and no visual field defect was detected using confrontation testing and Goldmann perimetry. Her left optic disc was swollen and hyperaemic, and her blood pressure was 160/70 mmHg. Incipient NA-AION was diagnosed and brimonidine tartrate $0.15 \%$ eye drops (Alphagan ${ }^{\circledR}$, Allergan Inc, Texas, USA) were prescribed twice daily. At followup five weeks later, there was complete resolution of the optic disc swelling without any pallor or atrophy, and Goldmann visual fields were also normal.

\section{Case 2}

A 56-year-old Chinese woman presented to the Accident and Emergency (A\&E) department of Singapore General Hospital on August 15, 2012 with complaints of flashing lights, left blurred vision $(\mathrm{VL}=6 / 6)$ and headaches that persisted for three weeks. She was under medical treatment for hypertension. Examination at the A\&E department revealed a swollen left optic disc, which was initially thought to be due to papilloedema. This resulted in the patient being admitted into a neurology ward, where she underwent a series of investigations, including magnetic resonance $(M R)$ imaging of the brain and postnasal space, and a lumbar puncture. The tests results were all negative. Eight days later, prior to her discharge from the neurology ward, the patient was referred to our centre. We found a left RAPD and hyperaemic swollen optic disc, but no visual field defect was detected on confrontation testing. Both Goldmann perimetry and Humphrey visual field analysis were unremarkable. She was diagnosed with incipient NA-AION and Alphagan ${ }^{\circledR}$ eye drops were prescribed. Over a period of three months, the left optic disc swelling gradually resolved without pallor or atrophy and repeat Humphrey visual field analysis was unremarkable.

\section{Case 3}

A 42-year-old Chinese man with no past medical history presented to a private eye clinic on November 19, 2012, with a two-week history of floaters. On examination, the left optic disc was found to be swollen, his blood pressure was $136 / 79 \mathrm{mmHg}$ and the erythrocyte sedimentation rate was $1 \mathrm{~mm} / \mathrm{hr}$. Infective workup and blood lipids were normal. The following day, the patient presented to our centre. On examination, his vision was $6 / 6$, but a left RAPD was present. Confrontation visual field examination, Goldmann perimetry and Humphrey visual field analysis were all unremarkable. The patient's left optic disc was swollen and hyperaemic (Fig. 1a). Incipient NA-AION was diagnosed and Alphagan ${ }^{\circledR}$ eye drops were prescribed. MR imaging of the brain and orbits (performed later in a different institution) was unremarkable. Follow-up at our centre three months after diagnosis showed complete resolution of the optic disc swelling with no pallor or atrophy (Fig. 1b). Repeat Humphrey visual field analysis did not show any defect.

\section{Case 4}

A 63-year-old Chinese woman with diabetic retinopathy and hypertension was referred to us from one of our retina/diabetic clinics on March 11, 2013. She complained of a three-week history of vague further loss of vision in her right eye. Her vision was reduced to counting fingers due to diabetic maculopathy and there was no RAPD recorded. Despite previous laser treatments, her Goldmann fields were full. However, her right optic disc was swollen and hyperaemic. Blood pressure recorded earlier was 133/80 mmHg. Alphagan ${ }^{\circledR}$ eye drops were prescribed on the presumptive diagnosis of incipient NA-AION. The optic disc swelling remained unchanged after four weeks of treatment, but completely resolved after another six weeks of treatment. There was no pallor or atrophy, and repeat Goldmann fields were still full. Formerly, the diagnosis for this patient would likely have been diabetic papillopathy.

\section{Case 5}

A 60-year-old Chinese woman with a known history of hypertension presented to our clinic on April 2, 2013 with a 
four-week history of flashes and floaters in the right eye. On examination, $V R=6 / 7.5$; there was no RAPD and confrontation visual field examination and Humphrey visual field analysis were normal. The patient's right optic disc was swollen and hyperaemic, and her blood pressure was 150/80 $\mathrm{mmHg}$. Incipient NA-AION was diagnosed and Alphagan ${ }^{\circledR}$ eye drops were prescribed. Follow-up at two months showed complete resolution of the optic disc swelling with no pallor or atrophy. Repeat Humphrey visual field analysis and Goldmann perimetry were unremarkable.

\section{DISCUSSION}

Although the condition described earlier has been termed 'incipient NA-AION', the term 'threatened NA-AION' may be more apt, as functional loss does not always occur. A swollen optic nerve head with loss of visual acuity points to a diagnosis of anterior optic neuritis (i.e. papillitis), while a small optic disc and visual field loss (especially when it involves the inferior fields) suggest possible ischaemic optic neuropathy. Bilateral swollen discs with normal visual function should raise the suspicion of papilloedema and raised intracranial pressure. As described earlier, the so-called incipient NA-AION is asymptomatic and characterised by unilateral optic disc swelling and normal relevant modalities of visual function (namely best corrected visual acuity, colour vision and visual field examination), unless another condition such as diabetic maculopathy is present, as was observed in Case 4. Typically, spontaneous resolution with gradual lessening of the optic disc swelling occurs without the development of optic disc pallor or atrophy. A similar variety of optic disc oedema with spontaneous resolution occurs in the so-called diabetic papillopathy, which was originally described in young persons with type 1 diabetes mellitus and later, in older persons with type 2 diabetes mellitus; this condition is now generally accepted to be a mild form of NA-AION. ${ }^{(4)}$

The only comprehensive paper on incipient NA-AION in the literature is a study published in 2007 by Hayreh and Zimmerman from lowa, USA..$^{(3)}$ In this study, out of a total of 54 patients (mean age 58.7 years), 34 (63\%) had diabetes mellitus, ${ }^{(3)}$ whereas only one out of five of our patients were diabetic $(20 \%)$, which is surprising in view of the high incidence of diabetes mellitus in the adult population in Singapore. On the other hand, only onethird of the patients from the American study had hypertension, while $80 \%$ of our patients $(n=4)$ were hypertensive and receiving treatment. While $55 \%$ of the patients from the American study had classical NA-AION in the fellow eye, with $25 \%$ of the eyes progressing from incipient to classical disease in a median time of 5.8 weeks, none of our patients had previous NA-AION in the fellow eye and none progressed to the classical NA-AION stage. Compared to the American study, our overall median time to resolution was longer ( 9.6 vs. 5.8 weeks), although it was comparable to the median time to resolution among their nonprogress cases. Also, corticosteroids were given to the majority of patients in the American study, whereas only Alphagan ${ }^{\circledR}$ eye drops were prescribed to our patients because it is not a practice in our centre to use corticosteroids for the treatment of incipient or classical NA-AION.

To conclude, the most important question is whether there is any effective treatment for incipient and classical NA-AION, and in particular, whether there are any measures that can be taken to prevent visual loss in incipient NA-AION or second-eye involvement in patients with established single-eye disease. As the pathogenesis of NA-AION is now accepted to be a perfusion problem involving the ciliary circulation that supplies the optic nerve head, ${ }^{(5)}$ any measure to improve or sustain circulation would be a logical approach to treatment. Thus, avoiding a sudden drop in systemic blood pressure, prescribing antihypertensive treatment to be taken only early in the day and lowering the intraocular pressure even if it is normal is our recommended management. All five of our patients were prescribed brimonidine tartrate eye drops twice daily to be used for as long as optic disc oedema persisted, this drug having both intraocular pressure-lowering and neuroprotective actions, and as noted above, none of our patients so treated progressed to full-blown NA-AION.

\section{ADDENDUM}

In the three months after this paper was accepted for publication (total study time therefore one year), we encountered two more Chinese patients with incipient NA-AION - one male aged 61 and one female aged 58 , both with multiple vascular risk factors. Neither had a RAPD and optic disc swelling in both patients resolved spontaneously after four and five weeks, respectively. They were also treated with Alphagan ${ }^{\circledR}$ eye drops for the duration of their disc swelling.

\section{REFERENCES}

1. Cullen JF, Por YM. Ischaemic optic neuropathy: the Singapore scene. Singapore Med J 2007; 48:281-6.

2. Cullen JF, Chung SH. Non-arteritic anterior ischaemic optic neuropathy (NA-AION): outcome for visual acuity and visual field defects, the Singapore scene 2. Singapore Med J 2012; 53:88-90

3. Hayreh SS, Zimmerman MB. Incipient nonarteritic anterior ischemic optic neuropathy. Ophthalmology 2007; 114:1763-72.

4. Arnold AC. Ischemic optic neuropathy. In: Miller NR, Newman NJ, eds. Walsh \& Hoyt's Clinical Neuro-Ophthalmology. 6th ed. Philadelphia Lippincott Williams \& Wilkins, 2005: 377-9.

5. Hayreh SS. Ischemic optic neuropathies. Heidelberg: Springer, 2011: 265-308. 